\title{
Pneumothorax leading to pneumopericardium after transvenous lead implantation in a patient with a previous epicardial lead
}

\author{
Raghav Bansal ${ }^{1}$, Ankit Mahajan ${ }^{2}$, Chetan Rathi $^{2}$, and Yash Lokhandawala ${ }^{3}$ \\ ${ }^{1}$ All India Institute of Medical Sciences \\ ${ }^{2}$ Holy Family Hospital \\ ${ }^{3}$ Arrhythmia Associates
}

September 11, 2020

\begin{abstract}
A 44-year-old lady, a follow up case of idiopathic dilated cardiomyopathy and cardiac resynchronization therapy defibrillator (CRT-D) device implantation with epicardial left ventricular (LV) lead, underwent a transvenous LV lead revision in view of epicardial lead malfunction. A chest X-ray after this, done for worsening dyspnoea, revealed pneumopericardium along with left pneumothorax. The CT revealed a communication between the left pleural and pericardial cavities, around the old epicardial lead. Drainage of the left pleural cavity resolved both the pneumothorax and pneumopericardium and the patient remained well on follow up.
\end{abstract}

Pneumothorax leading to pneumopericardium after transvenous lead implantation in a patient with a previous epicardial lead

Raghav Bansal, MD, DM¹; Ankit Mahajan, MD, DM² ; Chetan Rathi, MD, DNB ${ }^{2}$;

Yash Lokhandwala, MD, $\mathrm{DM}^{3}$

${ }^{1}$ Assistant Professor, Cardiology, All India Institute of Medical Sciences, New Delhi, India

${ }^{2}$ Electrophysiology Fellow, Holy Family Hospital, Bandra West, Mumbai, India

${ }^{3}$ Consultant, Holy Family Hospital, Bandra West, Mumbai, India

Address for Correspondence:

Dr Raghav Bansal

Room number $32,7^{\text {th }}$ floor

Department of Cardiology, CN Centre

All India Institute of Medical Sciences, New Delhi - 110029

India

Correspondence e-mail:raghav.mamc@gmail.com

Keywords: Cardiac resynchronisation, heart failure, pneumothorax, pneumopericardium, chest CT scan,chest X-ray

Word Count (excluding abstract, references and figure legends): 890

Financial disclosures: None 
There are no conflicts of interest

\begin{abstract}
A 44-year-old lady, a follow up case of idiopathic dilated cardiomyopathy and cardiac resynchronization therapy defibrillator (CRT-D) device implantation with epicardial left ventricular (LV) lead, underwent a transvenous LV lead revision in view of epicardial lead malfunction. A chest X-ray after this, done for worsening dyspnoea, revealed pneumopericardium along with left pneumothorax. The CT revealed a communication between the left pleural and pericardial cavities, around the old epicardial lead. Drainage of the left pleural cavity resolved both the pneumothorax and pneumopericardium and the patient remained well on follow up.
\end{abstract}

\title{
Introduction
}

Pneumothorax after pacemaker implantation is a well-known complication, reported in less than $1 \%$ of cases. ${ }^{1}$ The pneumothorax occurs as a complication of transvenous access. Pneumopericardium along with pneumothorax has been rarely reported to occur after pacemaker implantation. With a left sided implantation, the mechanism of pneumopericardium is different when it is associated with a right pneumothorax vis a vis a left pneumothorax. In this report, we present an interesting case with a novel mechanism of pneumopericardium associated with left pneumothorax.

\section{Case}

A 44-year-old lady was admitted with worsening heart failure symptoms for 1 month. She was a diagnosed case of idiopathic dilated cardiomyopathy with severe left ventricular (LV) dysfunction and had undergone cardiac resynchronization therapy defibrillator (CRT-D) device implantation 1 year ago, at another centre. At that time, the LV lead implantation could not be performed by the transvenous route due to a large iatrogenic coronary sinus dissection and an epicardial LV lead was subsequently implanted. She responded well initially, but after 6 months, her symptoms recurred. Device interrogation revealed epicardial lead failure, necessitating a lead revision. At this time she was referred to us.

We planned to implant the LV lead via the transvenous route. A left coronary angiogram in the venous washout phase revealed a normally flowing coronary sinus without any evidence of residual dissection. A left arm venogram showed patent left axillary and subclavian veins. The patient underwent a coronary sinus lead implantation successfully and the epicardial lead was capped and sutured to the pocket. Of note, the pocket had many adhesions and the left axillary/subclavian vein puncture was particularly difficult,necessitating multiple attempts.

A good electrocardiographic result with narrowing of QRS complex, prominent R wave in lead V1 and superior QRS axis were obtained. However, the patient complained of deteriorating dyspnoea the next day. Examination was suggestive of left pneumothorax with diminished breath sounds and tympanic note on percussion. Chest X-ray confirmed the diagnosis of a large left pneumothorax and surprisingly also revealed a pneumopericardium. A left pleural drian was immediately placed(Figure 1a). Transthoracic echocardiography was inconclusive due to a poor window, but the Doppler derived hemodynamics were not suggestive of cardiac tamponade. To help clarify the cause of pneumopericardium a CT scan of the chest was performed, which confirmed the presence of pneumopericardium, along with extrathoracic emphysema(Figure 2). It further revealed a connection between the left pleural and pericardial cavities around the epicardial lead, which allowed air to track from one to the other. The chest X-ray three days after intercostal drainage revealed resolution of pneumopericardium and pneumothorax (Figure 1b) and the patient was subsequently discharged. The patient continued to do well on follow up after 6 months.

\section{Discussion}

Pneumopericardium may occur due to multiple etiologies, which can be broadly classified as iatrogenic, inflammatory (pericarditis), fistulous (with adjacent air containing organs) and traumatic. Iatrogenic causes include pericardiocentesis, thoracocentesis, laparoscopy, cardiac electronic device implantation, sternal bone 
marrow aspiration and esophagoscopy. Pneumopericardium occurring after device implantation is rare, with literature limited to anecdotal case reports. The mechanisms to explain development of pneumopericardium after device implantation have been variously proposed on a case to case basis. ${ }^{2-8}$ Perforation of the helix of the right atrial lead into the right pleural cavity through the atrial wall and pericardium can cause pneumopericardium with right pneumothorax, especially with active fixation leads being implanted in thin, frail and elderly patients. ${ }^{2-5}$ This is the most common mechanism reported for pneumopericardium after CIED implantation. ${ }^{2-5}$ The association of pneumopericardium with left pneumothorax which occurs as a complication of subclavian venous puncture has been hypothesized to occur secondary to presence of a pleuro-pericardial connection. Chen et al reported partial absence of the left pericardium as the cause of such a pleuro-pericardial connection. ${ }^{6}$ It has been hypothesized that pleuro-pericardial micro-fistulae may form in patients who have undergone previous cardiac surgery. ${ }^{7-8}$ Thus in patients with previous history of cardiac surgery, these connections can then track air and consequently result in a pneumopericardium in case a pneumothorax occurs. In the present case, a connection between the left pleural cavity and the pericardium was created by the placement of the epicardial lead, as demonstrated conclusively by the CT scan. This is an important surgical consideration, as this situation may arise if adequate precautions are not observed to retract lung tissue during placement and tunneling of an epicardial lead.

Once a diagnosis of pneumopericardium is made, cardiac tamponade must be carefully excluded. Transthoracic echocardiography may be tricky due to an inadequate echo window, secondary to air inside the pericardium. The hemodynamics and clinical picture are helpful in this regard. An elevated jugular venous pressure with absent y descent, low cardiac output with hypotension, paradoxical pulse and distant heart sounds help in making a clinical diagnosis of cardiac tamponade, requiring immediate relief with pericardial drainage. If there are no signs of tamponade, drainage of the pneumothorax with an inter-costal drainage tube is all that required to treat both pneumopericardium and pneumothorax due to the presence of connection between the two cavities, as in this case.

To conclude, pneumopericardium is a rare complication occurring after CIED implantation. A CT scan helps delineating the mechanism of pneumopericardium. In a patient with previous epicardial lead implantation, the possibility of the lead creating a pleuro-pericardial connection should be considered. After ruling out cardiac tamponade, drainage of the pneumothorax is adequate to drain both air spaces.

\section{References}

1. Kirkfeldt RE, Johansen JB, Nohr EA, Moller M, Arnsbo P, Nielsen JC. Pneumothoraxin cardiac pacing: a population-based cohort study of 28,860 Danishpatients. Europace 2012;14(8):1132e8.

2. Srivathsan K, Byrne RA, Appleton CP, Scott LRP. Pneumopericardium and pneumothorax contralateral to venous access site after permanent pacemakerimplantation. Europace 2003;5(4):361e3.

3. Sebastian CC, Wu W-C, Shafer M, Choudhary G, Patel PM. Pneumopericardium and pneumothorax after permanent pacemaker implantation. Pacing ClinElectrophysiol 2005;28(5):466e8.

4. Nantsupawat T, Li JM, Benditt DG, Adabag S. Contralateral pneumothorax and pneumopericardium after dual-chamber pacemaker implantation: Mechanism, diagnosis, and treatment. HeartRhythm Case Rep. 2018;4(6):256-259.

5. Parashar NK, Siddharthan D, Yadav R, Sinha M, Ramakumar V. An unexpected complication of intracardiac device implantation:Contralateral pneumothorax and pneumopericardium. Indian Pacing Electrophysiol J. 2019;19(4):167-170.

6. Chen J, Tsai Y, Li Y. Unusual pneumopericardium during permanent pacemaker implantation. Heart 2011; 97: 2094.

7. Parahuleva M, Schifferings P, Neuhof C, Tillmanns H, Erdogan A. Pneumopericardium and pneumomediastinum as a late complication of defibrillator implantation after coronary artery bypass graft surgery. Thorac Cardiovasc Surg. 2009;57(8):491-3.

8. Green PG, Herring N. Pneumopericardium and pneumomediastinum after implantationof a cardiac resynchronization pacemaker. JACC Case Rep. 2019;1(3):381-4.

\section{Figure legends}


Figure 1. a. Chest X-ray frontal view soon after left intercostal tube drainage, showing pneumopericardium (bold arrow) and left pneumothorax. b. Chest X-ray frontal view after 3 days, demonstrating complete resolution of pneumothorax and pneumopericardium.

Figure 2. Axial section of HRCT chest demonstrating epicardial LV lead puncturing the pericardium (bold arrow) through the left pleural cavity, making a communication between the two. Also note, air in the pericardial cavity seen on the $\mathrm{CT}$ scan image and the presence of extrathoracic emphysema secondary to intercostal drainage.
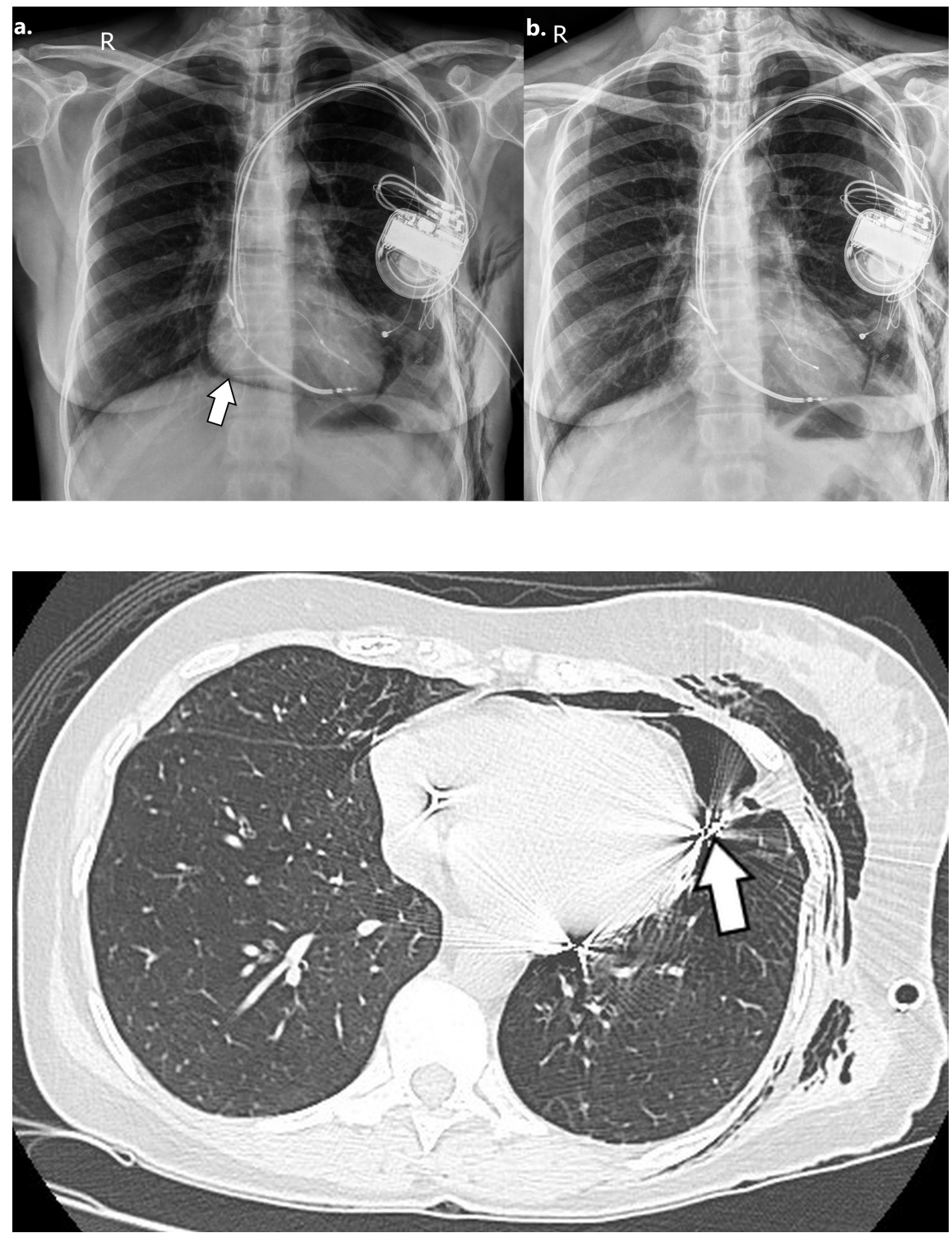